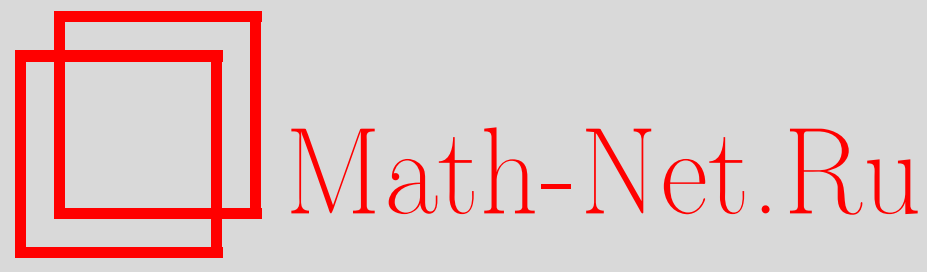

О. В. Пугачев, Емкости и поверхностные меры в локально выпуклых пространствах, Теория вероятн. и ее примен., 2008, том 53, выпуск 1, 178-189

DOI: https://doi.org/10.4213/tvp2493

Использование Общероссийского математического портала Math-Net.Ru подразумевает, что вы прочитали и согласны с пользовательским соглашением

http: //www.mathnet.ru/rus/agreement

Параметры загрузки:

IP : 52.23 .180 .231

26 апреля 2023 г., 15:42:11

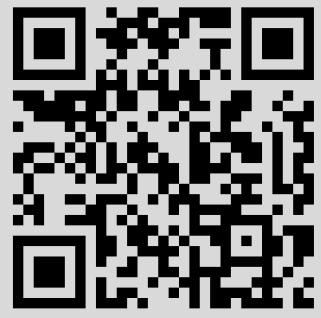


4. Ибрагимов P., Шарахметов Ш. Точная константа в неравенстве Розенталя для случайных величин с нулевым средним. - Теория вероятн. и ее примен., 2001, т. 46 , в. 1 , с. $134-138$.

5. Пешкир Г., Ширяев А.Н. Неравенства Хинчина и мартингальное расширение сферы их действия. - Успехи матем. наук, 1995, т. 50, № 5, с. 3-62.

Поступила в редакцию 15.VIII.2006

(C) 2008 г

ПУГАЧЕВ О.В.*

\section{ЕМКОСТИ И ПОВЕРХНОСТНЫЕ МЕРЫ В ЛОКАЛЬНО ВЫПУКЛЫХ ПРОСТРАНСТВАХ ${ }^{1)}$}

Доказывается плотность емкостей, порожденных соболевскими классами любого порядка в широком классе локально выпуклых пространств. Эти емкости применяются при построении поверхностных мер на множествах уровня соболевских функций и локальных соболевских функций.

Ключевые слова и фразы: дифференцируемая мера, соболевские классы в локально выпуклых пространствах, плотность емкости, поверхностная мера, формула Остроградского-Гаусса, локальные соболевские функции.

1. Введение. Поверхностные меры и емкости в бесконечномерных пространствах представляют интерес как для самой аналитической и геометрической теории меры, так и для ее приложений в нелинейном анализе, в теории случайных процессов, в теории линейных дифференциальных уравнений относительно функций и мер.

Первый подход к построению поверхностных мер в бесконечномерных пространствах берет начало в книге А. В. Скорохода [1], затем он был существенно развит в работах А. В. Угланова [2]-[4]. Он основан на построении локальных поверхностных мер в достаточно малых окрестностях точек поверхности.

Совершенно другой подход, реализованный Э. Эро и П. Маллявэном в работе [5] для винеровской меры и В.И. Богачевым [6]-[8] для дифференцируемых мер, в дальнейшем был развит автором. В этом методе мера строится сразу на всей поверхности, а условия гладкости поверхности связаны не с геометрией объемлюшего пространства, а с геометрией подпространства Камерона-Мартина, причем функция, задаюшая поверхность, принадлежит соболевскому классу, она даже не обязана быть непрерывной (таковы типичные функции, появляющиеся в теории случайных процессов).

В приложениях иногда встречаются функции, не являющиеся соболевскими глобально, но обладающие подобными свойствами локально. Цель данной работы обобщить результаты, полученные в [9], [10], для локальных соболевских функций и задаваемых ими поверхностей.

Новизна данной работы по сравнению с [9]-[11] также и в том, что результаты получены для более широкого класса локально выпуклых пространств.

2. Соболевские классы. Пусть $X-$ локально выпуклое пространство; пусть сепарабельное гильбертово пространство $H$ непрерывно вложено в $X$. Обозначим через $\langle\cdot ; \cdot\rangle$ скалярное произведение в $H$, через $|\cdot|$ - норму в $H$. Тогда формула

$$
j_{H}: X^{*} \rightarrow H^{*}=H, \quad\left\langle j_{H}(l) ; h\right\rangle=l(h) \quad \forall h \in H,
$$

* Московский государственный технический университет им. Н.Э. Баумана, 2-я Бауманская ул., 5, 105005 Москва, Россия; e-mail: opugachev@yandex.ru

1) Работа выполнена при поддержке грантов РФФИ 07-01-00536 и DFG 436 RUS 113/343/0(R). 
задает непрерывное отображение с плотным множеством значений. Если функция $f$ дифференцируема в точке $x \in X$ в каком-либо смысле (Гато, Фреше или Соболева), то вектор $D_{H} f(x)=j_{H}\left(f^{\prime}(x)\right)$ (градиент вдоль $H$ ) соответствует линейному функционалу $f^{\prime}(x) \in X^{*}$.

Если $\mu$ - мера на $X$ и функция $g$ является $\mu$-измеримой, то $g \cdot \mu$ будет обозначать меру с плотностью $g$ относительно $\mu$.

Пусть $E$ - сепарабельное гильбертово пространство. Обозначим $\mathscr{H}_{1}(H, E)$ класс операторов Гильберта-Шмидта из $H$ в $E$, наделенный нормой Гильберта-Шмидта

$$
\|T\|_{\mathscr{H}_{1}(H, E)}^{2}=\sum_{m=1}^{\infty}\left\|T e_{m}\right\|_{E}^{2}
$$

где $\left\{e_{m}\right\}$ - ортонормированный базис в $H$. Классы операторов Гильберта-Шмидта высших порядков определяются индуктивно:

$$
\mathscr{H}_{n}(H, E)=\mathscr{H}_{1}\left(H, \mathscr{H}_{n-1}(H, E)\right), \quad n=2,3, \ldots,
$$

они наделены нормами

$$
\|T\|_{\mathscr{H} e_{n}(H, E)}^{2}=\sum_{m_{1}=1}^{\infty} \cdots \sum_{m_{n}=1}^{\infty}\left\|T\left(e_{m_{1}}, \ldots, e_{m_{n}}\right)\right\|_{E}^{2} .
$$

При $n=0$ естественно положить $\mathscr{H}_{0}(H, E)=E$.

Функция $f: X \rightarrow E$ называется гладкой цилиндрической, если она имеет вид

$$
f(x)=\sum_{k=1}^{n} u_{k}\left(l_{1}(x), \ldots, l_{m}(x)\right) y_{k}
$$

где $n, m \in \mathbf{N}, u_{k} \in C_{b}^{\infty}\left(\mathbf{R}^{m}\right), l_{j} \in X^{*}, y_{k} \in E$. Обозначим класс таких функций $\mathscr{F} \mathscr{C}_{b}^{\infty}(X, E)$. Легко видеть, что любая гладкая цилиндрическая функция $f$ непрерывна и бесконечно дифференцируема по Фреше; ее градиент $r$-го порядка вдоль $H$ принадлежит $\mathscr{H}_{r}(H, E)$ в любой точке $x \in X$, и его норма Гильберта-Шмидта $\left\|D_{H}^{r} f(x)\right\|_{\mathscr{H}_{r}(H, E)}$ равномерно ограничена.

Всюду далее в случае $E=\mathbf{R}$ мы будем опускать индекс $E$ в обозначениях классов функций.

О п р е д е л е н и е 2.1. Мера $\mu$ на $X$ дифференцируема в смысле Фомина вдоль вектора $h \in X$, если сушествует знакопеременная мера $d_{h} \mu$ такая, что для любого борелевского множества $B \subset X$ имеем

$$
\left.\frac{d}{d t}\right|_{t=0} \frac{\mu(B+t h)-\mu(B)}{t}=d_{h} \mu(B) .
$$

Известно (см. [7], [8]), что мера $d_{h} \mu$ абсолютно непрерывна по мере $\mu$. Соответствующая плотность Радона-Никодима обозначается $\beta_{h}^{\mu}$ и называется логарифмической производной меры $\mu$ вдоль вектора $h$.

Для негауссовской вероятностной меры $\mu$ на локально выпуклом пространстве $X$ введем следующий аналог пространства Камерона-Мартина:

$$
H(\mu)=\left\{h \in X: \beta_{h}^{\mu} \in L^{2}(\mu)\right\} .
$$

Это пространство оказывается гильбертовым с нормой $\|h\|_{H(\mu)}=\left\|\beta_{h}^{\mu}\right\|_{L^{2}(\mu)}$; оно непрерывно вложено в $X$; если $L^{2}(\mu)$ сепарабельно, то сепарабельно и $H(\mu)(\mathrm{cm} .[8$, гл. 5]).

Далее будем предполагать, что мера $\mu$ такова, что если функция $\varphi \in \mathscr{F}_{b}^{\infty}(X)$ равна нулю $\mu$-п.в., то $\mu$-п.в. $D_{H} \varphi=0$. В частности, это верно в случае $\operatorname{supp} \mu=X$. Дополнительно предположим, что

$$
j_{H}\left(X^{*}\right) \subset H(\mu) .
$$

Мера $\mu$ дифференцируема (в смысле Фомина) вдоль векторного поля $v: X \rightarrow X$, если существует $\mu$-интегрируемая функция $\delta v$ (дивергенция $v$ ) такая, что

$$
\int \partial_{v} \varphi(x) \mu(d x)=-\int \varphi(x) \delta v(x) \mu(d x) \quad \forall \varphi \in \mathscr{F} \mathscr{C}_{b}^{\infty}(X) .
$$


О п р е д е л е н и е 2.2. Функция $f \in L^{p}(\mu, E)$ входит в соболевский класс $W^{r, p}(\mu, E), p \geqslant 1, r \in \mathbf{N}$, если сушествует последовательность функций $f_{n} \in$ $\mathscr{F} \mathscr{C}_{b}^{\infty}(X, E)$, сходяшаяся к $f$ в $L^{p}(\mu, E)$ и фундаментальная по норме

$$
\|f\|_{r, p, E}=\|f\|_{L^{p}(\mu, E)}+\sum_{k=1}^{r}\left\|D_{H}^{k} f\right\|_{L^{p}\left(\mu, \mathscr{H}_{k}(H, E)\right)} .
$$

Для $m=1, \ldots, r$ предел в $L^{p}\left(\mu, \mathscr{H}_{m}(H, E)\right)$ функций $D_{H}^{m} f_{n}$ называется грациентом порядка $m$ функции $f$ и обозначается символом $D_{H}^{m} f$.

Если выполнено условие (1), то при $p \geqslant 2$ соболевские градиенты не зависят от выбора последовательности $\left\{f_{n}\right\}$.

Доказательства следующих четырех лемм можно найти в [8] и [10].

Лемма 2.1. (i) Ecлu $f \in W^{r, p}(\mu), g \in W^{r, q}(\mu), 1<p, q<\infty, 1 / p+1 / q=1 / s \leqslant 1$, mо $f g \in W^{r, s}(\mu)$, причем $\|f g\|_{r, s} \leqslant 2^{r}\|f\|_{r, p}\|g\|_{r, q}$.

(ii) Ecлu $f \in W^{r, p}(\mu, H), h \in W^{r, q}(\mu, H)$ dлs $r \geqslant 0,1 / p+1 / q=1 / s \leqslant 1$, mo $\langle f, h\rangle \in W^{r, s}(\mu)$.

Лемма 2.2. Пусть $f \in W^{r, p}(\mu) ;$ пусть $\varphi \in C_{b}^{r-1}(\mathbf{R})$ такова, что $\varphi^{(r-1)}$ липиицева. Тогда функиия $\varphi \circ f \in W^{r, p}(\mu)$ и ее градиенты порядка от 1 до $r$ выражаются по иепному правилу:

$D_{H}(\varphi \circ f)=\varphi^{\prime} \circ f \cdot D_{H} f, \quad D_{H}^{2}(\varphi \circ f)=\varphi^{\prime \prime} \circ f \cdot D_{H} f \otimes D_{H} f+\varphi^{\prime} \circ f \cdot D_{H}^{2} f \quad$ u m.d.

Лемма 2.3. Пусть $f \in W^{1, p}(\mu)$. Если мера $\mu$ дифферениируема вдоль векторного поля $v \in L^{q}(\mu, H), \delta v \in L^{q}(\mu), 1 / p+1 / q=1$, то мера $f \mu$ также дифференцируема вдоль $v$, причем $d_{v}(f \mu)=\left(f \cdot \delta v+\partial_{v} f\right) \mu$.

Лемма 2.4. Если $j_{H}\left(X^{*}\right) \subset H(\mu)$, то соболевские классы $W^{r, p}(\mu), p \geqslant 2$, обладают свойством локальности, т.е. если бункиия $f$ принадлежит к одному из таких классов и $A=\{f=0\}$, то все производнье $f$ порядков $k \leqslant r$ обращаются в нуль u-n.в. на $A$.

3. Емкости. Пусть $\mathscr{F}-$ некоторый класс функций, наделенный такой нормой $\|\cdot\|_{0}$, что если $\mu$-п.в. $f=0$, то $\|f\|_{0}=0$. Емкость, порожденная $\mathscr{F}$, определяется следуюшим образом.

О п р е д е л е н и е 3.1. Если множество $U \subset X$ открыто, то

$$
C_{\mathscr{F}}(U)=\inf \left\{\|f\|_{0}: f \in \mathscr{F}, f \geqslant 0 ; f \geqslant 1 \text { на } U \mu \text {-п.в. }\right\} .
$$

Для произвольного множества $A \subset X$ положим

$$
C_{\mathscr{F}}(A)=\inf \left\{C_{\mathscr{F}}(U): A \subset U, U \text { открыто }\right\} .
$$

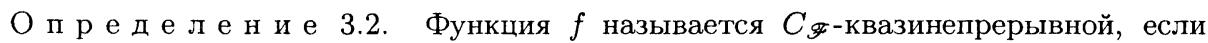
существуют такие замкнутые множества $Q_{1} \subset Q_{2} \subset \cdots$, что $f$ непрерывна на каждом $Q_{n}$ и $C_{\mathscr{F}}\left(X \backslash Q_{n}\right)<1 / n$.

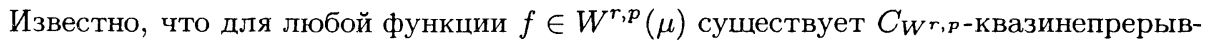
ная $\mu$-версия (см. $[8$, теорема 7.4.6]). Очевидно, что для любого множества $A \subset X$ верны оценки $C_{W^{r, p}}(A) \leqslant C_{W^{r^{\prime}, p^{\prime}}}(A)$ при $p \leqslant p^{\prime}, r \leqslant r^{\prime}$.

О п р е д е л е н и е 3.3. Пусть $H$ - сепарабельное гильбертово пространство, непрерывно вложенное в $X$. Будем говорить, что $H$ удовлетворяет условию (T1), если существует центрированная радоновская гауссовская мера $\gamma$ на $X$ такая, что $H \subset H(\gamma)$ (это вложение автоматически непрерывно).

Лемма 3.1. Пусть $H \subset X-$ сепарабельное гильбертово пространство, удовлетворяющее (Т1). Пусть $f: X \rightarrow \mathbf{R}$ - ограниченная борелевская функиия. Тогда функиия

$$
F(x)=\int_{X} f(x+y) \gamma(d y)
$$

бесконечно много раз дифферениируема по Гато вдоль $H$, ее производные $D_{H}^{n} F(x)$ являются операторами Гильберта-Шмидта и

$$
\left\|D_{H}^{n} F(x)\right\|_{\mathscr{H}_{n}(H)} \leqslant c_{n}\|f(x+\cdot)\|_{L^{2}(\gamma)} \leqslant c_{n} \sup _{y}|f(y)|
$$

где $c_{n}=c^{n} \sqrt{n !}$ и с есть норма оператора вложения $H \hookrightarrow H(\gamma)$. 
Д о к а з а т е л ь с т в о. Пусть $H_{0}$ - замыкание $H$ в $H(\gamma)$, наделенное нормой из пространства $H(\gamma)$. Гильбертово пространство $H_{0}$ сепарабельно, как и $H$. Поскольку вложение $H \hookrightarrow H(\gamma)$ имеет ограниченную норму $c$, получаем

$$
\|T\|_{\mathscr{H}_{n}(H, E)} \leqslant c^{n}\|T\|_{\mathscr{H}_{n}\left(H_{0}, E\right)}
$$

для любого оператора Гильберта-Шмидта $T: H_{0} \rightarrow E$. Следовательно, достаточно доказать наше утверждение в случае равенства норм $H$ и $H_{0}$. Сушествование производных Гато следует из [8, пример 2.1.15]. Пусть $\left\{e_{j}\right\}$ - ортонормированный базис $H=H_{0}$. Зафиксируем порядки дифференцирования $k_{1}, k_{2}, \ldots$ вдоль $e_{1}, e_{2}, \ldots$ соответственно; $k_{1}+\cdots+k_{m}=n$. Обозначим $L_{m}=\left\{\sum_{j=1}^{m} t_{j} e_{j} \mid t_{j} \in \mathbf{R}\right\} ; X=X_{m} \oplus L_{m}$. Тогда $\gamma$ представима в виде произведения гауссовских мер на $X_{m}$ и на $L_{m}$. Имеем

$$
\gamma=\gamma_{m} \otimes\left(\frac{1}{(2 \pi)^{m / 2}} \exp \left[-\frac{1}{2} \sum_{j=1}^{m} t_{j}^{2}\right] d t_{1} \cdots d t_{m}\right) .
$$

Разложив $y=z+\sum_{j=1}^{m} t_{j} e_{j}$, находим

$$
\begin{aligned}
\partial_{e_{1}}^{k_{1}} \cdots \partial_{e_{m}}^{k_{m}} F(x)= & \partial_{t_{1}}^{k_{1}} \cdots \partial_{t_{m}}^{k_{m}} \int_{X_{m}} \int_{L_{m}} f\left(x+z+\sum_{j=1}^{m} t_{j} e_{j}\right) \\
& \times \frac{1}{(2 \pi)^{m / 2}} \exp \left[-\frac{1}{2} \sum_{j=1}^{m} t_{j}^{2}\right] d t_{1} \cdots d t_{m} \gamma_{m}(d z) \\
= & \int_{X_{m}} \int_{L_{m}} f\left(x+z+\sum_{j=1}^{m} t_{j} e_{j}\right) \prod_{j=1}^{m}\left(\partial_{t_{j}}^{k_{j}} \frac{\exp \left(-t_{j}^{2} / 2\right)}{\sqrt{2 \pi}}\right) d t_{1} \cdots d t_{m} \gamma_{m}(d z) \\
= & (-1)^{n} \int_{X} f\left(x+z+\sum_{j=1}^{m} t_{j} e_{j}\right) \prod_{j=1}^{m}\left(\sqrt{k_{j} !} H_{k_{j}}\left(t_{j}\right)\right) \gamma(d y),
\end{aligned}
$$

где $H_{k}(t), k=0,1,2, \ldots$, - полиномы Эрмита. Известно (см. [7]), что функции $\prod_{j=1}^{m} H_{k_{j}}\left(t_{j}\right)$ ортонормированы в $L^{2}(\gamma)$. По неравенству Бесселя

$$
\begin{aligned}
& \sum_{i_{1}=1}^{\infty} \cdots \sum_{i_{n}=1}^{\infty}\left(\partial_{e_{i_{1}}} \cdots \partial_{e_{i_{n}}} F(x)\right)^{2} \\
& \quad=\sum_{m=1}^{\infty} \sum_{k_{1}+\cdots+k_{m}=n}\left(\int_{X} f(x+y) \prod_{j=1}^{m}\left(\sqrt{k_{j} !} H_{k_{j}}\left(t_{j}\right)\right) \gamma(d y)\right)^{2} \\
& \quad \leqslant n ! \int_{X} f(x+y)^{2} \gamma(d y)=n !\|f(x+\cdot)\|_{L^{2}(\gamma)}^{2} \leqslant n ! \cdot \sup _{y}|f(y)|^{2} .
\end{aligned}
$$

Лемма 3.1 доказана.

Следствие 3.1. Для функиии $F$, построенной в лемме 3.1 , при каждом $p \geqslant 2$ мы имеем

$$
\|F\|_{L^{p}(\mu)} \leqslant\|f\|_{L^{p}(\mu * \gamma)}, \quad\left\|D_{H}^{n} F\right\|_{L^{p}\left(\mu, \mathscr{H}_{n}(H)\right)} \leqslant c_{n}\|f\|_{L^{p}(\mu * \gamma)}
$$

Д ок а з а те л ь с т в о. Функция $F$ является борелевской (см. [13, лемма 7.6.4]). Пусть $A \subset X-\mu$-измеримое множество. Определим меру

$$
\mu_{F}(A)=\int_{A} \int f(x+y) \gamma(d y) \mu(d x) \equiv \int f(z)\left(\left(\left.\mu\right|_{A}\right) * \gamma\right)(d z) .
$$

Мера $\mu_{F}$ абсолютно непрерывна относительно $\mu$, и ее плотность совпадает с $F \mu$-п.в. Пусть $p \geqslant 2$. Тогда

$$
\int|F(x)|^{p} \mu(d x) \leqslant \int\|f(x+\cdot)\|_{L^{1}(\gamma)}^{p} \mu(d x)
$$




$$
\begin{aligned}
& \leqslant \int\|f(x+\cdot)\|_{L^{p}(\gamma)}^{p} \mu(d x)=\|f\|_{L^{p}(\mu * \gamma)}^{p}, \\
\int\left\|D_{H}^{n} F(x)\right\|_{\mathscr{H}(H)}^{p} \mu(d x) & \leqslant c_{n}^{p} \int\|f(x+\cdot)\|_{L^{2}(\gamma)}^{p} \mu(d x) \\
& \leqslant c_{n}^{p} \int\|f(x+\cdot)\|_{L^{p}(\gamma)}^{p} \mu(d x)=\left(c_{n}\|f\|_{L^{p}(\mu * \gamma)}\right)^{p}
\end{aligned}
$$

что завершает доказательство.

О п р е д е л е н и е 3.4 . Емкость $C_{\mathscr{F}}$ плотна, если для любого $\varepsilon>0$ существует компакт $K_{\varepsilon} \subset X$ такой, что $C_{\mathscr{F}}\left(X \backslash K_{\varepsilon}\right)<\varepsilon$.

Первый положительный результат о плотности соболевских емкостей в негауссовском случае -- следующая теорема (см. [12, предложение 3.1]).

Теорема 3.1. Пусть $X$ - сепарабельное банахово пространство с вероятностной мерой $\mu$, и пусть $H$ - сепарабельное гильбертово пространство, непрерывно вложенное в $X$. Предположим, что соболевский класс $W^{1, p}(\mu)$ корректно определен. Тогда емкость $C_{W^{1, p}(\mu)}$ плотна.

Следуюший результат данной статьи является новым.

Теорема 3.2. Пусть $X$ - локально выпуклое пространство с радоновской вероятностной мерой $\mu$. Предположим, что для любого $\varepsilon>0$ существует метризуемый компакт $K^{\varepsilon} \subset X$ такой, что $\mu\left(X \backslash K^{\varepsilon}\right)<\varepsilon$. Пусть $H \subset X-$ cenapaбельное гильбертово пространство, для которого выполнено (Т1). Тогда емкость $C_{r, p}=C_{W^{r, p}(\mu)}$ плотна при любых $p \in[1 ;+\infty), r \in \mathbf{N}$, при которых соболевский класс $W^{r, p}(\mu)$ определен корректно.

Д о к а з а т е л ь с т в о. Выберем центрированную радоновскую гауссовскую меру $\gamma$ такую, что $H(\gamma) \supset H$. Положим $\varepsilon=10^{-j}$ для $j \in \mathbf{N}$; пусть $K^{\varepsilon}$ - метризуемый компакт с $\mu\left(X \backslash K^{\varepsilon}\right)<\varepsilon$, и пусть $K_{\gamma}^{\varepsilon}-$ абсолютно выпуклый метризуемый компакт с $\gamma\left(X \backslash K_{\gamma}^{\varepsilon}\right)<\varepsilon$. Обозначим через $K_{j}$ выпуклую оболочку множества $K^{\varepsilon} \cup\left(-K^{\varepsilon}\right) \cup K_{\gamma}^{\varepsilon}$.

Рассмотрим функцию $\varphi_{j}(x)=\gamma\left(X \backslash\left(2 K_{j}-x\right)\right)$. Если $x \in K_{j}$, то $2 K_{j}-x \supset K_{j}$, отсюда $\varphi_{j}(x) \leqslant \gamma\left(X \backslash K_{j}\right)<\frac{1}{10}$. С другой стороны, если $x \notin 2 K_{j}$, то

$$
1-\varphi_{j}(x)=\gamma\left(2 K_{j}-x\right)=\gamma\left(-2 K_{j}+x\right)
$$

но компакты $2 K_{j}-x$ и $-2 K_{j}+x$ не пересекаются: иначе нашлась бы точка $y$ такая, что $y+x \in 2 K_{j},-y+x \in 2 K_{j}$, тогда $x \in 2 K_{j}$. Следовательно, $\varphi_{j}(x) \geqslant \frac{1}{2}$.

Функция $\varphi_{j}$ имеет вид (3) с $f(x)=I_{X \backslash 2 K_{j}}(x)$. По лемме 3.1 функция $\varphi_{j}$ дифференцируема по Гато сколько угодно раз вдоль любых векторов $h \in H$, и мы получаем оценку

$$
\left\|D_{H}^{n} \varphi_{j}(x)\right\|_{H} \leqslant c_{n}\left\|I_{X \backslash\left(2 K_{j}-x\right)}\right\|_{L^{2}(\gamma)},
$$

где $c_{n}=c^{n} \sqrt{n !}, c-$ норма оператора вложения $H \hookrightarrow H(\gamma)$.

Зафиксируем неубывающую функцию $\psi \in C^{\infty}(\mathbf{R})$, равную 0 на $\left[0 ; \frac{1}{10}\right]$ и 1 на $\left[\frac{1}{2} ; 1\right]$. Положим $g_{j}=\psi \circ \varphi_{j}$. Тогда $1 \geqslant g_{j} \geqslant 0,\left.g_{j}\right|_{X \backslash 2 K_{j}}=1,\left.g_{j}\right|_{K_{j}}=0$, и мы получаем

$$
\left\|g_{j}\right\|_{L^{p}(\mu)} \leqslant\left(\mu\left(X \backslash K_{j}\right)\right)^{1 / p} \longrightarrow 0
$$

при $j \rightarrow \infty$. Кроме того, оценив градиенты функции $g_{j}$ по цепному правилу, получаем

$$
\begin{aligned}
\int\left\|D_{H}^{n} g_{j}(x)\right\|_{H}^{p} \mu(d x) & \leqslant\left(c_{n} \cdot 2^{n} \sup _{[0 ; 1]} \sum_{k=1}^{n}\left|\psi^{(k)}\right|\right)^{p} \int\left\|I_{X \backslash\left(2 K_{j}-x\right)}\right\|_{L^{2}(\gamma)}^{p} \mu(d x) \\
& \leqslant C_{n}(p)\left[\int_{X \backslash K_{j}} \mu(d x)+\int_{K_{j}}\left(\gamma\left(X \backslash K_{j}\right)\right)^{p / 2} \mu(d x)\right] \\
& \leqslant C_{n}(p)\left(10^{-j}+10^{-j p / 2}\right) \rightarrow 0 \quad \text { при } j \rightarrow \infty .
\end{aligned}
$$

Следовательно, $C_{r, p}\left(X \backslash 2 K_{j}\right) \leqslant\left\|g_{j}\right\|_{r, p}<\varepsilon$ для достаточно больших $j$.

Для завершения доказательства того, что $g_{j} \in W^{r, p}$, надо построить последовательность гладких цилиндрических функций, сходящуюся к $g_{j}$ в $L^{p}(\mu)$ и фундаментальную по норме $\|\cdot\|_{r, p}$. 
Пусть $Y$ обозначает линейную оболочку $\bigcup_{j=1}^{\infty} K_{j}$. Линейное подмногообразие $Y$ является суслинским пространством, поэтому существует непрерывный инъективный линейный оператор $I$, отображающий $Y$ в сепарабельное пространство Фреше $\Phi=\mathbf{R}^{\infty}$. Образ $K_{j}$ под действием $I$ есть абсолютно выпуклый компакт $Q \subset \Phi$. Он представим в виде счетного пересечения замкнутых полупространств вида $\left\{l_{n} \leqslant 1\right\}, l_{n} \in \Phi^{*}$. Следовательно, сушествует последовательность убывающих замкнутых выпуклых цилиндров $C_{n}=\bigcap_{i=1}^{n}\left\{l_{i} \leqslant 1\right\}$ таких, что $\bigcap_{n=1}^{\infty} C_{n}=Q$. Линейные функционалы $l_{i} \circ I: X \rightarrow \mathbf{R}$ непрерывны на $X$. Пусть

$$
B_{n}=\left\{x \in X: l_{i}(I(x)) \leqslant 1, i=1, \ldots, n\right\} ;
$$

тогда $\bigcap_{n=1}^{\infty} B_{n} \cap Y=K_{j}$. Рассмотрим функции

$$
f_{n}(x)=\gamma\left(X \backslash\left(2 B_{n}-x\right)\right) .
$$

Легко проверить, что $f_{n}$ имеет вид

$$
f_{n}(x)=u\left(l_{1}(x), \ldots, l_{n}(x)\right), \quad u \in C_{b}^{\infty}\left(\mathbf{R}^{n}\right) .
$$

Следовательно, $f_{n} \in \mathscr{F} \mathscr{C}_{b}^{\infty}(X)$. Для всех $x \in Y$, а значит, для $\mu$-почти всех $x \in X$ имеем $f_{n}(x) \rightarrow \varphi_{j}(x)$, откуда благодаря равномерной ограниченности функций $f_{n}$ по теореме Лебега следует, что $\left\|f_{n}-\varphi_{j}\right\|_{L^{p}(\mu)} \rightarrow 0$. Пусть $n>m$. По следствию 3.1 получаем

$$
\left\|D_{H}^{n} f_{n}-D_{H}^{n} f_{m}\right\|_{L^{p}(\mu, H)} \leqslant C_{n}\left\|I_{B_{m} \backslash B_{n}}\right\|_{L^{\max \{p ; 2\}}(\mu * \gamma)} \longrightarrow 0
$$

равномерно по $n>m$ при $m \rightarrow \infty$. Итак, $\left\{f_{n}\right\}$ - аппроксимирующая последовательность для $\varphi_{j}$. Наконец, $\left\{\psi \circ f_{n}\right\} \subset \mathscr{F}_{b}^{\infty}(X)-$ аппроксимируюшая последовательность для $g_{j}=\psi \circ \varphi_{j}$. Теорема 3.2 доказана.

3 а м е ч а н и е 3.1. Доказательства в этой статье остаются в силе, если условие (Т1), накладываемое на гильбертово подпространство $H$, заменить следующим условием (Т): существует чентрально-симметричная радоновская мера $\lambda$ на $X$ та$\kappa a$, что $H \subset H(\lambda)$. Это условие несколько слабее, чем (T1), однако во многих пространствах эти два условия эквивалентны (например, если $X$ гильбертово, ядерное, $\mathscr{S}$ или $\mathscr{S}^{\prime}$ ). Лишь доказательство леммы 3.1 в этом случае становится длиннее, cM. [11].

\section{4. Поверхностные меры.}

Теорема 4.1. Пусть $X$ - локально выпуклое пространство с радоновской мерой $\mu$ (возможно, знакопеременной), и пусть $H$ - сепарабельное гильбертово пространство, непрерывно вложенное в $X$. Если функиия $F: X \rightarrow \mathbf{R}$ обладает следуюшими свойствами:

a) $F \in W^{2, p}(\mu), p>4$,

b) $\left|D_{H} F\right|^{-1} \in L^{p}(\mu)$,

c) $\mu$ дифФеренцируема вдоль векторного поля $v=D_{H} F$ c $\delta v \in L^{2}(\mu)$, то образ меры $\mu \circ F^{-1}$ абсолютно непрерывен относительно мерь Лебега на $\mathbf{R}$, причем его плотность $k$ имеет непрерывную версию с ограниченной вариачией.

Д о к а з а т е л ь с т в о. Для доказательства существования требуемой плотности покажем, что сушествует мера $\lambda_{1}$ на $X$ такая, что для любой функции $\varphi \in C_{b}^{1}(\mathbf{R})$

$$
\int \varphi^{\prime}(t) \mu \circ F^{-1}(d t) \equiv \int \varphi^{\prime}(F(x)) \mu(d x)=-\int \varphi(F(x)) \lambda_{1}(d x)
$$

откуда, согласно [8, предложение 2.2.3, (ii)], будет вытекать дифференцируемость меры $\mu \circ F^{-1}$ по Скороходу. Сделаем следующие преобразования:

$$
\begin{aligned}
\int \varphi^{\prime}(F(x)) \mu(d x) & =\int \varphi^{\prime}(F(x)) \partial_{v} F(x) \frac{\mu(d x)}{\partial_{v} F(x)}=\int \partial_{v}(\varphi(F(x))) \frac{\mu(d x)}{\left|D_{H} F(x)\right|^{2}} \\
& =-\int \varphi(F(x)) \cdot d_{v}\left(\frac{\mu}{\left|D_{H} F\right|^{2}}\right)(d x) .
\end{aligned}
$$

Чтобы обосновать последнее равенство, заметим, что $\left|D_{H} F\right|^{2} \in W^{1, p / 2}(\mu)$ в силу леммы 2.1 , следовательно, функции $G_{n}=\left(\left|D_{H} F\right|^{2}+1 / n\right)^{-1}$ принадлежат тому же 
классу по лемме 2.2. При этом $G_{n} \nearrow\left|D_{H} F\right|^{-2} \in L^{p / 2}(\mu)$, откуда $G_{n} \rightarrow\left|D_{H} F\right|^{-2}$ по норме $L^{p / 2}$. Кроме того,

$$
D_{H} G_{n}=\frac{-D_{H}\left\langle D_{H} F, D_{H} F\right\rangle}{\left(\left|D_{H} F\right|^{2}+1 / n\right)^{2}}=\left(\frac{\left|D_{H} F\right|^{2}}{\left|D_{H} F\right|^{2}+1 / n}\right)^{2} D_{H}\left|D_{H} F\right|^{-2},
$$

где первый сомножитель возрастает, стремясь к 1 при $\mu$-почти всех $x$, а норма второго (не зависящего от $n$ ) сомножителя не превосходит

$$
\frac{2\left|D_{H}^{2} F\left(D_{H} F\right)\right|}{\left|D_{H} F\right|^{4}} \leqslant \frac{2\left\|D_{H}^{2} F\right\| \mathscr{H}_{2}(H)}{\left|D_{H} F\right|^{3}} \in L^{p / 4}(\mu),
$$

следовательно, функция $\left|D_{H} F\right|^{-2}=\lim _{n \rightarrow \infty} G_{n}$ принадлежит классу $W^{1,2}(\mu)$. В силу леммы 2.3 мера $\left|D_{H} F\right|^{-2} \mu$ дифференцируема вдоль векторного поля $v$, причем ее производная равна

$$
\lambda_{1}=d_{v}\left(\frac{\mu}{\left|D_{H} F\right|^{2}}\right)=\left(-\frac{2 D_{H}^{2} F\left(D_{H} F, D_{H} F\right)}{\left|D_{H} F\right|^{4}}+\frac{\delta v}{\left|D_{H} F\right|^{2}}\right) \mu .
$$

Функция $\varphi \circ F$ ограничена и по лемме 2.2 принадлежит классу $W^{1, p}(\mu)$, поэтому мы можем выбрать последовательность равномерно ограниченных гладких цилиндрических функций $f_{n}$, сходяшихся к $\varphi \circ F$ по норме $\|\cdot\|_{1, p}$ и $\mu$-почти всюду. Тогда в формуле интегрирования по частям

$$
\int \partial_{v} f_{n}(x) \frac{\mu}{\left|D_{H} F\right|^{2}}(d x)=-\int f_{n}(x) \lambda_{1}(d x)
$$

можно перейти к пределу по $n$ (по теореме Лебега) и получить соответствующую формулу для функции $\varphi \circ F$. Равенство (5) доказано.

Таким образом, мера $\mu \circ F^{-1}$ дифференцируема на $\mathbf{R}$ в смысле Скорохода; $d_{1}\left(\mu \circ F^{-1}\right)=\lambda_{1} \circ F^{-1}$. Из этого следует (см. [8, пример 2.2 .1 , (ii)]), что некоторая версия лебеговской плотности $k(t)$ меры $\mu \circ F^{-1}$ имеет конечную вариацию (не превышаюшую $\left.\left\|\lambda_{1}\right\|\right)$.

Но поскольку $\lambda_{1} \ll \mu$, мы получаем $d_{1}\left(\mu \circ F^{-1}\right) \ll\left(\mu \circ F^{-1}\right)$, следовательно, мера $\mu \circ F^{-1}$ на прямой дифференцируема также и в смысле Фомина, а для меры $d_{1}\left(\mu \circ F^{-1}\right)$ сушествует плотность $k^{\prime}$ относительно меры Лебега, причем

$$
k(t)=\int_{-\infty}^{t} k^{\prime}(u) d u \quad \forall t \in \mathbf{R} .
$$

В частности, функция $k$ непрерывна. Теорема 4.1 доказана.

Применив теорему 4.1 к мере $g \mu$ вместо $\mu$, получаем следующее утверждение.

Следствие 4.1. Предположим, что выполнены условия теоремы 4.1. Пусть

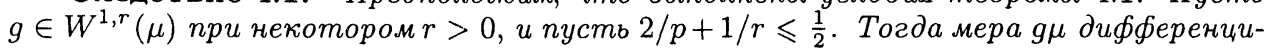
руема вдоль $v=D_{H} F$, причем ее образ $(g \mu) \circ F^{-1}$ имеет непрерывную плотность $k_{g}$, вариация которой не превосходит const $\cdot\|g\|_{1, r}$.

Следующие две теоремы были доказаны в [10], причем $X$ предполагалось сепарабельным пространством Фреше. Однако теперь теоремы остаются в силе и для более общего случая, благодаря результатам п. 3.

Теорема 4.2. Пусть $X$ - локально выпуклое пространство, $\mu$ - вероятностная мера, сосредоточенная на последовательности метризуемьх компактов, $u$ пусть $H \subset X$ - сепарабельное гильбертово пространство, удовлетворяющее (Т1). Пусть $F \in W^{2,8}(\mu),\left|D_{H} F\right|^{-1} \in L^{8}(\mu)$. Пусть мера $\mu$ диФФерениируема вдоль векторного поля $v=D_{H} F$, причем $\delta v \in L^{2}(\mu)$. Тогда существует единственная радоновская мера $\nu$ со свойством

$$
\int \varphi(x) \nu(d x)=k_{\varphi}(0) \quad \forall \varphi \in \mathscr{F} \mathscr{C}_{b}^{\infty}(X)
$$

Мера $\nu$ равна нулю на множествах нулевой емкости $C_{1, r}$ при $r$ таких, что

$$
\frac{2}{p}+\frac{1}{r}<\frac{1}{2}
$$


Пусть теперь мера $\mu$ неотрицательна. Введем дополнительные обозначения. Пусть $\nu^{(0)} \equiv \nu$ и $\nu^{(a)}$ - меры, построенные по теореме 4.2 для функции $F-a$ вместо $F$; положим $\mu_{\sigma}^{(a)}=\nu^{(a)} / k(a)$; если $k(a)=0$, положим $\mu_{\sigma}^{(a)} \equiv 0$.

Теорема 4.3. Пусть выполнены условия теоремь 4.2. Пусть $F$ является $C_{1,4}$ квазинепрерывной. Тогда мерьц $\mu_{\sigma}^{(a)}$ являются условньми мерами для $\mu$ относительно функиии $F$, т.е.:

1) при $\mu \circ F^{-1}-$-поти всех а меры $\mu_{\sigma}^{(a)}$ вероятностные и сосредоточеньл на поверхностях $F^{-1}(a)$;

2) для любого борелевского $B \subset X$ имеем $\mu(B)=\int_{-\infty}^{+\infty} \mu_{\sigma}^{(a)}(B) \mu \circ F^{-1}(d a)$.

5. Поверхностные меры, порожденные локальными соболевскими функциями. Пусть $\mu$ - вероятностная мера на локально выпуклом пространстве $X$.

О п р е д е л е н и е 5.1. Пусть $\mathscr{K}$ - некоторое счетное семейство компактов на $X$. Назовем $\mathscr{K}$ локализующим $r$-го порядка, если

1) для любых $K_{1}, K_{2} \in \mathscr{K}$ сушествует $K_{3} \in \mathscr{K}$ такой, что $K_{1} \cup K_{2} \subset K_{3}$,

2) $\mu\left(X \backslash \bigcup_{K \in \mathscr{K}} K\right)=0$,

3) для каждого $K \in \mathscr{K}$ существует такая $C_{r, p}$-квазинепрерывная при всех $p$ функция $\zeta_{K} \in W^{r, \infty}(\mu)=\bigcap_{p} W^{r, p}(\mu)$, что $0 \leqslant \zeta_{K} \leqslant 1,\left.\zeta_{K}\right|_{K}=1$, и существует такой компакт $K^{\prime} \in \mathscr{K}$, что $\left.\zeta_{K}\right|_{X \backslash K^{\prime}}=0$.

Семейство функций $\left\{\zeta_{K} \mid K \in \mathscr{K}\right\}$ будем также называть локализующим порядка $r$.

Лемма 5.1. Пусть $\mathscr{K}=\left\{K_{i}\right\}$ - возрастаюиая последовательность метризуемьх абсолютно выпукльх компактов такая, ито $\mu\left(X \backslash \bigcup_{K \in \mathscr{K}} K\right)=0$; для каждого $K \in \mathscr{K}$ сушествует $K^{\prime} \in \mathscr{K}$ такой, что $2 K \subset K^{\prime}$. Тогда последовательность компактов $\mathscr{K}$ - локализуюшее семейство любого порядка $r \in \mathrm{N}$, если $H$ вложено в $H(\gamma)$ для некоторой радоновской чентрированной гауссовской меры $\gamma u$ $\gamma\left(X \backslash \bigcup_{K \in \mathscr{K}} K\right)=0$.

Д о к а з а т е л ь с т в о. Из условий следует, что емкости $C_{r, p}$ плотны при всех $r \in \mathbf{N}, p \geqslant 1$. Зафиксируем $r \in \mathbf{N}$. Возьмем функцию $g=1-g_{j}$, где $g_{j}$ - функция, построенная в доказательстве теоремы 3.2 , такая, что $g_{j}(x)=1$ при $x \notin 2 K, g_{j}(x)=0$ при $x \in K$ и $g_{j} \in W^{r, p}$ для всех $p \geqslant 1$. Для функции $g$ можно выбрать $\mu$-версию, $C_{r, p}$-квазинепрерывную для всех $p$ (см. $[11$, следствие 5.2]). Тогда мы можем взять $K^{\prime} \supset 2 K$. Лемма 2.3 доказана.

О п р е д е л е н и е 5.2. Будем говорить, что функция $f$ принадлежит классу $W_{\text {Loc }}^{r, p}(\mu), p>2$, если сушествует локализуюшее семейство функций $\left\{\zeta_{K}\right\} r$-го порядка такое, что

$$
\zeta f \in \bigcap_{q<p} W^{r, q}(\mu) \quad \forall \zeta \in\left\{\zeta_{K}\right\} .
$$

Производные $f$ в точке $x \in K \in \mathscr{K}$ определяются по формуле

$$
D_{H}^{k} f(x)=D_{H}^{k}\left(\zeta_{K} f\right)(x), \quad k=1, \ldots, r .
$$

В силу леммы 2.4 при выборе другого локализующего семейства эта величина останется $\mu$-почти всюду неизменной.

Из леммы 2.1 следует, что при любых $r$ и $p$ имеем $W^{r, p}(\mu) \subset W_{\mathrm{Loc}}^{r, p}(\mu)$.

Лемма 5.2. Пусть $f \in W_{\mathrm{Loc}}^{k, p}(\mu), g \in W_{\mathrm{Loc}}^{k, q}(\mu), 1 / p+1 / q=1 / s<\frac{1}{2}$. Тогда

1) $f g \in W_{\mathrm{Loc}}^{k, s}(\mu)$

2) ecлu $k>0$, mo $\left\langle D_{H} f ; D_{H} g\right\rangle \in W_{\text {Loc }}^{k-1, s}(\mu)$.

Д о к а з а т е л ь с т в о. Пусть $\left\{\zeta_{n}^{f}\right\}$ - локализуюшее семейство функций порядка $k$ для $f$, а $\left\{\zeta_{n}^{g}\right\}$ - такое же семейство для $g$. Тогда функции $\zeta_{n}:=\zeta_{n}^{f} \zeta_{n}^{g}$ образуют локализующее семейство для обеих функций $f$ и $g$. Зафиксируем $\zeta=\zeta_{n}$. Зададим $s^{\prime} \in(2 ; s)$. Существует функция $\xi=\zeta_{N}$, равная 1 на $\operatorname{supp} \zeta$. Тогда $\zeta \equiv \zeta \xi$.

1) Поскольку $\zeta f g \equiv \zeta f \cdot \xi g$, из $\zeta f \in W^{k, p s^{\prime} / s}(\mu)$ и $\xi g \in W^{k, q s^{\prime} / s}(\mu)$ по лемме 2.1 следует, что $\zeta f g \in W^{k, s^{\prime}}(\mu)$. 
2) Функция $\zeta\left\langle D_{H} f ; D_{H} g\right\rangle$, в силу свойства локальности почти всюду совпадающая с $\zeta\left\langle D_{H}(\xi f) ; D_{H}(\xi g)\right\rangle$, принадлежит $W^{k-1, s^{\prime}}(\mu)$ согласно лемме 2.1, поскольку

$$
D_{H}(\xi f) \in W^{k-1, p s^{\prime} / s}(\mu, H), \quad D_{H}(\xi g) \in W^{k-1, q s^{\prime} / s}(\mu, H) .
$$

Лемма 2.4 доказана.

Теперь обобщим теорему 4.1 на случай локальных соболевских классов.

Теорема 5.1. Пусть $\mu-$ радоновская вероятностная мера на локально выпуклом пространстве $X, H$ - сепарабельное гильбертово пространство, $H \subset H(\mu)$. Пусть $F \in W_{\mathrm{Loc}}^{2, p}(\mu),\left|D_{H} F\right|^{-1} \in L_{\mathrm{Loc}}^{p}(\mu) \equiv W_{\mathrm{Loc}}^{0, p}(\mu)$, где $p>4$. Предnоложим, что существует локализующее семейство функиий $\mathscr{L} 2$-го порядка для $F$ и $\left|D_{H} F\right|^{-1}$. Если для любой функиии $\zeta \in \mathscr{L}$ мера $\zeta \mu$ дифференцируема вдоль векторного поля $D_{H} F$, причем плотность $\rho_{\zeta}$ меры $d_{D_{H}}(\zeta \mu)$ относительно меры $\mu$ принадлежит $L^{2}(\mu)$, то мера $(\zeta \mu) \circ F^{-1}$ имеет непрерьвную плотность $k^{\zeta}$ (относительно меры Лебега) с ограниченной вариачией.

Д о к а з а т е ль с т в о. Пусть $\zeta, \xi \in \mathscr{L}$ таковы, что $\xi=1$ на носителе функции $\zeta$. Тогда $\xi\left|D_{H} F\right|^{2} \in W^{1,2+\varepsilon}(\mu)$. Следовательно, функции $G_{n}=\xi^{2} /\left(\xi\left|D_{H} F\right|^{2}+1 / n\right)$ принадлежат классу $W^{1,2}(\mu)$ ввиду леммы 2.1 и [8, лемма 7.1.13]. Применив те же рассуждения, что и в теореме 4.1, получаем, что функция $\xi\left|D_{H} F\right|^{-2}=\lim _{n \rightarrow \infty} G_{n}$ принадлежит классу $W^{1,2}(\mu)$. Следовательно, по лемме 2.3 мы получаем меру

$$
\lambda_{\zeta}=d_{D_{H} F}\left(\frac{\xi \zeta \mu}{\left|D_{H} F\right|^{2}}\right)=\left(-\frac{\partial_{D_{H} F}^{2} F}{\left|D_{H} F\right|^{4}} \zeta+\frac{\rho_{\zeta}}{\left|D_{H} F\right|^{2}}\right) \mu .
$$

Ее образ $\lambda_{\zeta} \circ F^{-1}$ на $\mathbf{R}$ является производной в смысле Фомина от меры $(\zeta \mu) \circ F^{-1}$, откуда следует сушествование искомой плотности. Теорема 5.1 доказана.

Лемма 5.3. Пусть $f \in W_{\mathrm{Loc}}^{r, p}(\mu)$, тогда при любом $p^{\prime}<p$ функиия $f$ имеет $C_{r, p^{\prime}}$-квазинепрерывную версию, если выполнено следующее условие: $\inf _{K \in \mathscr{K}} C_{r, p^{\prime}}(X \backslash$ $K)=0$.

Д о к а з а т е л ь с т в о. Зафиксируем $p^{\prime}<p$. Выберем последовательность компактов $K_{n} \in \mathscr{K}$ и функций $\zeta_{n}=\zeta_{K_{n}}$ со свойствами: $\operatorname{supp} \zeta_{n} \subset K_{n+1}, C_{r, p^{\prime}}(X \backslash$ $\left.K_{n}\right)<2^{-n}$. Положим $\xi_{1}=\zeta_{1} ; \xi_{n}=\zeta_{n}-\zeta_{n-1}$ при $n>1$. Выберем для функции $\zeta_{n+1} f \in W^{r, p^{\prime}}(\mu)$ версию $g_{n}$, которая является $C_{r, p^{\prime}}$-квазинепрерывной. Тогда $f_{n}:=$ $\xi_{n} g_{n}=\xi_{n} f \mu$-почти всюду; $f_{n}$ равна 0 на $K_{n-1}$ и $C_{r, p^{\prime}}$-квазинепрерывна, т.е. можно выбрать такие замкнутые множества $Q_{n}^{m}, m \in \mathrm{N}$, что $Q_{n}^{m} \subset Q_{n}^{m+1}, C_{r, p^{\prime}}\left(X \backslash Q_{n}^{m}\right)<$ $2^{-n-m}$ и $f_{n}$ непрерывна на каждом $Q_{n}^{m}$. Положим $f_{o}(x)=\sum_{n=1}^{\infty} f_{n}(x)$. На $K_{n}$ эта сумма имеет лишь $n$ ненулевых слагаемых, и $\mu$-почти всюду $f_{o}=\lim _{n \rightarrow \infty} \zeta_{n} f=f$. Кроме того, при каждом $m \in \mathbf{N}$ функция $f_{o}$ непрерывна на замкнутом множестве

$$
F^{m}=\bigcap_{n=1}^{\infty} Q_{n}^{m} \cap K_{m} ; \quad F^{m} \subset F^{m+1} ; \quad C_{r, p^{\prime}}\left(X \backslash F^{m}\right)<2^{1-m} .
$$

Таким образом, $f_{o}$ - искомая версия $f$. Лемма 5.3 доказана.

Следуюшая теорема обобщает теоремы 4.2 и 4.3.

Теорема 5.2. Пусть $X$ - локально выпуклое пространство и выполнень условия теоремы 5.1. Тогда однозначно определено семейство радоновских мер $\left\{\nu_{\zeta}^{(a)} \mid \zeta \in \mathscr{L}, a \in \mathbf{R}\right\} \operatorname{ma\kappa ux,~umo~}$

$$
\int \varphi(x) \nu_{\zeta}^{(a)}(d x)=k_{\varphi}^{\zeta}(a) \quad \forall \varphi \in \mathscr{F} \mathscr{C}_{b}^{\infty}(X)
$$

Эти меры обращаются в нуль на множествах нулевой емкости $C_{1, r}$, где $r$ удовлетворяет условию (7).

Кроме того, если $\inf _{K \in \mathscr{K}} C_{W^{1,2 r}}(X \backslash K)=0$ и для функции $g \in W_{\mathrm{Loc}}^{1,2 r+\varepsilon}(\mu)$ выбрана $C_{1,2 r}$-квазинепрерывная версия, то

$$
\int g(x) \nu_{\zeta}^{(a)}(d x)=k_{g}^{\zeta}(a)
$$


О п р ед е л ен и е 5.3. Пусть выполнены все условия теоремы 5.2. Пусть $A \in \mathscr{B}(X)$. Если $A \subset K$ для некоторого $K \in \mathscr{K}$ ( $\mathscr{K}$ - локализуюшее семейство компактов 2-го порядка для функций $F$ и $\left.\left|D_{H} F\right|^{-1}\right)$ и $\operatorname{supp} \zeta_{K} \subset K^{\prime} \in \mathscr{K}$, то положим

если $A$ произвольно, положим

$$
\nu^{(a)}(A)=\nu_{\zeta_{K}^{\prime}}^{(a)}(A)
$$

$$
\nu^{(a)}(A)=\sup \left\{\nu^{(a)}(A \cap K): K \in \mathscr{K}\right\} .
$$

Предложение 5.1. Функиии $\nu^{(a)}: \mathscr{B}(X) \rightarrow[0 ;+\infty]$ корректно определены $и$ являются радоновскими б-конечными положительньми мерами, конечными на компактах из $\mathscr{K}$.

Д оказ а т ел ь с т в о. 1) Пусть $A \subset K_{1}, A \subset K_{2} ; \operatorname{supp} \zeta_{K_{j}} \subset K_{j}^{\prime}, \xi_{j}=\zeta_{K_{j}^{\prime}}$, $j=1,2$. Тогда $\xi_{1}=\xi_{2}=1$ на $K_{1}^{\prime} \cap K_{2}^{\prime}$. Положим $\psi=\xi_{1} \xi_{2}$. Обозначим через $g$ функцию $\zeta_{K_{1}} \zeta_{K_{2}}$ (равную единице на $K_{1} \cap K_{2}$ и нулю вне $K_{1}^{\prime} \cap K_{2}^{\prime}$ ). Поскольку $\xi_{j} \geqslant \psi$ для $j=1,2$, то для всякого $B \in \mathscr{B}(X)$ мы имеем $\nu_{\xi_{j}}^{(a)}(B) \geqslant \nu_{\psi}^{(a)}(B)$, откуда следует, что

$$
\int g(x) \nu_{\xi_{j}}^{(a)}(d x) \geqslant \int g(x) \nu_{\psi}^{(a)}(d x), \quad j=1,2,
$$

и равенство возможно лишь при совпадении этих мер на множестве $\{g>0\}$, содержашем $A$. Поскольку $g \xi_{j} \equiv g \psi$, имеем $k_{g}^{\xi_{j}}(t)=k_{g}^{\psi}(t)$ для почти всех и, следовательно, в силу непрерывности обеих функций, для всех $t \in \mathbf{R}$, откуда по теореме 5.2 получаем

$$
\int g(x) \nu_{\xi_{j}}^{(a)}(d x)=k_{g}^{\xi_{j}}(a)=k_{g}^{\psi}(a)=\int g(x) \nu_{\psi}^{(a)}(d x) .
$$

Поэтому $\nu_{\xi_{1}}^{(a)}(A)=\nu_{\xi_{2}}^{(a)}(A)$, следовательно, мера $\nu^{(a)}$ определена корректно для множества $A \subset K \in \mathscr{K}$, а значит, и для произвольного множества.

2) Для любого $K \in \mathscr{K}$ радоновские меры $\left.\nu^{(a)}\right|_{K}=\left.\nu_{\zeta_{K^{\prime}}}^{(a)}\right|_{K}$ конечны. Автоматически следует $\sigma$-конечность и неотрицательность мер $\nu^{(a)}$.

3) Конечная аддитивность меры $\nu^{(a)}$ очевидна, также очевидна $\sigma$-аддитивность в случае $A=\bigcup_{n=1}^{\infty} A_{n} \subset K, K \in \mathscr{K}$. Проверим $\sigma$-аддитивность в общем случае. Пусть $A_{1}, A_{2}, \ldots$ - непересекающиеся борелевские множества, $\nu^{(a)}\left(A_{n}\right)<\infty, A=\bigcup_{n=1}^{\infty} A_{n}$; пусть $A_{n, 1} \subset A_{n, 2} \subset \cdots \subset A_{n}, A_{n, m}$ являются подмножествами компактов из $\mathscr{K}$; $\nu^{(a)}\left(A_{n, m}\right) \geqslant \nu^{(a)}\left(A_{n}\right)-2^{-n-m}$. Тогда $B_{N}=\bigcup_{n=1}^{N} A_{n, N}$ - подмножество компакта из $\mathscr{K}, B_{N} \subset A$ и $\nu^{(a)}\left(B_{N}\right) \nearrow \sum_{n=1}^{\infty} \nu^{(a)}\left(A_{n}\right) \leqslant+\infty$ при $N \rightarrow \infty$. Следовательно, $\nu^{(a)}(A) \geqslant \sum_{n=1}^{\infty} \nu^{(a)}\left(A_{n}\right)$. С другой стороны, если $B \subset A, B \subset K, K \in \mathscr{K}$, то

$$
\nu^{(a)}(B)=\sum_{n=1}^{\infty} \nu^{(a)}\left(B \cap A_{n}\right) \leqslant \sum_{n=1}^{\infty} \nu^{(a)}\left(A_{n}\right)
$$

откуда следует обратное утверждение для $\nu^{(a)}(A)$. Предложение 5.1 доказана.

Теперь мы можем получить несколько следствий из теоремы 5.2.

Следствие 5.1. Пусть выполнены все условия теоремы 4.2. Тогда меры $\nu^{(a)}$ и $\mu_{\sigma}^{(a)}$, построенные в этом пункте, будут совпадать с аналогичными мерами из n. 4 .

Следствие 5.2. Если $\mathscr{K}_{1}$ и $\mathscr{K}_{2}$ - два локализующих семейства компактов 2-го порядка, удовлетворяюших всем условиям теоремы 5.2, то построенные по ним мерь $\nu_{1}^{(a)}$ и $\nu_{2}^{(a)}$ совпадym.

Д о к а з а т е л ь с т в о. Построим локализующее семейство компактов

$$
\mathscr{K}_{3}=\mathscr{K}_{1} \cup \mathscr{K}_{2} \cup\left\{K_{1} \cup K_{2} \mid K_{1} \in \mathscr{K}_{1}, K_{2} \in \mathscr{K}_{2}\right\}
$$

с локализуюшими функциями вида $\zeta_{K_{1}}, \zeta_{K_{2}}$ и $\phi\left(\zeta_{K_{1}}+\zeta_{K_{2}}\right)$, где $\phi \in C_{b}^{\infty}(\mathbf{R})$ монотонна, $\phi(0)=0, \phi(t)=1$ при $t \geqslant 1$. Тогда равенства $\nu_{1}^{(a)}=\nu_{3}^{(a)}, \nu_{2}^{(a)}=\nu_{3}^{(a)}$ следуют из п. 1) доказательства предложения 5.1 и из оценок локализованных поверхностных мер через емкости. 
Следствие 5.3. Пусть выполнены все условия теоремы 5.2. Тогда при почти всех $a \in \mathbf{R}$ мера $\nu^{(a)}$ конечна, и для любых функиий $\varphi \in \mathscr{F}_{\mathscr{C}_{b}^{\infty}}(X)$ u $\theta \in L^{\infty}(\mathbf{R})$ верно равенство

$$
\int_{-\infty}^{+\infty} \theta(a) \cdot(\varphi \mu) \circ F^{-1}(d a)=\int_{-\infty}^{+\infty} \theta(a) \int \varphi(x) \nu^{(a)}(d x) d a .
$$

Д о к а з а т е л ь с т в о. Выберем произвольную последовательность $\left\{\zeta_{n}\right\} \subset \mathscr{L}$ такую, что $\zeta_{n} \nearrow 1$ в $L^{1}(\mu)$. Тогда для неотрицательной $\varphi \in \mathscr{F} \mathscr{C}_{b}^{\infty}(X)$ мы имеем

$$
\left\|\left(\zeta_{n} \varphi \mu\right) \circ F^{-1}-(\varphi \mu) \circ F^{-1}\right\| \leqslant\left\|\zeta_{n} \varphi \mu-\varphi \mu\right\| \longrightarrow 0 \quad \text { при } \quad n \rightarrow \infty .
$$

Функция $\varrho(t)=\lim _{n \rightarrow \infty} k_{\varphi}^{\zeta_{n}}(t)$ является $L^{1}(\mathbf{R})$-пределом функций $k_{\varphi}^{\zeta_{n}}$ и почти всюду конечна, поскольку в силу теоремы Б. Леви

$$
\int_{\mathbf{R}} \varrho(t) d t=\lim _{n \rightarrow \infty} \int_{\mathbf{R}} k_{\varphi}^{\zeta_{n}}(t) d t=\lim _{n \rightarrow \infty} \int_{X} \varphi \zeta_{n} d \mu \leqslant \int_{X} \varphi d \mu<\infty .
$$

Следовательно, ввиду $(9),(\varphi \mu) \circ F^{-1}(d t)=\varrho(t) d t$, поскольку предел мер по вариации единствен. С другой стороны, в силу (6)

$$
\int \varphi(x) \nu^{(a)}(d x)=\sup \left\{\int \varphi(x) \nu_{\zeta}^{(a)}(d x): \zeta \in \mathscr{L}\right\}=\sup \left\{k_{\varphi}^{\zeta}(a): \zeta \in \mathscr{L}\right\}=\varrho(a)
$$

для почти всех $a \in \mathbf{R}$. Следствие 5.3 доказана.

Формулу Остроградского-Гаусса (см. [9]) также можно обобшить для локально соболевских $F$ и $u$, см. [14].

Приведем пример, показываюший, что локализационный метод построения поверхностных мер действительно расширяет наши возможности.

П р и м е р 5.1. Пусть $X$ есть $\mathbf{R}^{\infty}$ с топологией покоординатной сходимости; $H=l^{2}$. Рассмотрим вероятностную меру

$$
\mu(d x)=\bigotimes_{n=1}^{\infty} \frac{d x_{n}}{\pi\left(1+x_{n}^{2}\right)} .
$$

Мера $\mu$ радоновская, поскольку $X$ - сепарабельное пространство Фреше. При этом $H(\mu)=l^{2}$ (см. [8, следствие 3.1.2]). Рассмотрим функцию $F(x)=\sum_{n=1}^{\infty} n^{-4} x_{n}^{2}$. Функции $F$ и $\left|D_{H} F\right|$ не интегрируемы (хотя конечны $\mu$-почти всюду). Построим семейство компактов

$$
\begin{gathered}
\mathscr{K}=\left\{K_{t}=\left\{x \in X:\left|x_{n}\right| \leqslant n^{5 / 4} t\right\} \mid t \in \mathbf{N}\right\} \\
\mu\left(K_{t}\right)=\prod_{n=1}^{\infty} \frac{2}{\pi} \operatorname{arctg}\left(n^{5 / 4} t\right) \geqslant 1-\sum_{n=1}^{\infty} \frac{2}{\pi n^{5 / 4} t} \longrightarrow 1 \quad \text { при } \quad t \rightarrow \infty .
\end{gathered}
$$

Семейство компактов $\mathscr{K}$ является локализующим любого порядка. Это следует из леммы 2.3, поскольку $H=H(\gamma)$, где $\gamma$ - счетное произведение стандартных гауссовских мер на прямых $\mathbf{R}$. Пусть $\mathscr{L}=\left\{\zeta_{K_{t}} \mid t \in \mathbf{N}\right\}-$ локализуюшие функции 2-го порядка, $\operatorname{supp} \zeta_{K_{t}} \subset K_{2 t}$. Имеем

$$
\mu\left\{x:\left|D_{H} F(x)\right|<\varepsilon\right\} \leqslant \mu\left\{\prod_{n=1}^{\infty}\left(-\frac{n^{4} \varepsilon}{2} ; \frac{n^{4} \varepsilon}{2}\right)\right\} \leqslant \prod_{n=1}^{p} \frac{n^{4} \varepsilon}{\pi}=\operatorname{const}(p) \cdot \varepsilon^{p}
$$

при любом $p \in \mathbf{N}$, следовательно, $\left|D_{H} F\right|^{-1} \in \bigcap_{p \geqslant 1} L^{p}(\mu)$.

При любом $t \in \mathrm{N}$ функции $F,\left|D_{H} F\right|$ и $\left\|D_{H}^{2} F\right\|_{\mathscr{H}(H)}$ ограничены на $K_{2 t}$ и при любом $p \geqslant 2$ функция $F$ приближается по норме $\|\cdot\|_{2, p}\left(\left.\mu\right|_{K_{2 t}}\right)$ цилиндрическими функциями $f_{N}(x)=\sum_{n=1}^{N} n^{-4} x_{n}^{2}$. Следовательно, для всех $\zeta \in \mathscr{L}$ имеем $\zeta F \in W^{2, \infty}(\mu)$. Мера $\zeta \mu$ дифференцируема вдоль векторного поля $D_{H} F$, поскольку в силу равномерной ограниченности $\beta_{e_{j}}^{\mu}$ ряд

$$
\sum_{j=1}^{\infty}\left(\partial_{e_{j}}^{2} F+\partial_{e_{j}} F \frac{\partial_{e_{j}} \zeta}{\zeta}+\partial_{e_{j}} F \cdot \beta_{e_{j}}^{\mu}\right) \zeta \mu
$$


сходится по вариации, и его сумма является мерой $d_{D_{H} F}(\zeta \mu)$, причем $\rho_{\zeta}=$ $d_{D_{H} F}(\zeta \mu) / \mu \in L^{2}(\mu)$. Следовательно, функция $F$, локализуюшее семейство функций $\mathscr{L}$ и мера $\mu$ удовлетворяют условиям теоремы 5.2 , и при всех $a>0$ сушествуют $\sigma$-конечные поверхностные меры $\nu^{(a)}$ и $\mu_{\sigma}^{(a)}$; очевидно, что при $a \leqslant 0$ они обращаются в нуль.

Поскольку $C_{1, p}\left(X \backslash K_{t}\right) \rightarrow 0$ при любом $p \geqslant 1$, справедлива формула (8) для $C_{1,4}$-квазинепрерывных $g \in W_{\text {Lоc }}^{1, r}(\mu)$ при $r>4$.

\section{СПИСОК ЛИТЕРАТУРЫ}

1. Скороход А. В. Интегрирование в гильбертовом пространстве. М.: Наука, 1975, $231 \mathrm{c}$.

2. Уаланов А.В. Поверхностные интегралы в линейных топологических пространствах. - Докл. РАН, 1995, т. 344, № 4, с. 450-453.

3. Угланов А.В. Поверхностные интегралы в пространствах Фреше. - Матем. сб., 1998 , т. 189 , № 11 , с. 139-157.

4. Uglanov A. $V$. Integration on infinite-dimensional surfaces and its applications. Dordrecht: Kluwer, 2000, $262 \mathrm{p}$.

5. Airault $H$., Malliavin P. Intégration géométrique sur l'espace de Wiener. - Bull. Sci. Math., 1988, v. 112, № 1, p. 3-52.

6. Bogachev V.I. Smooth measures, the Malliavin calculus and approximations in infinite dimensional space. - Acta Univ. Carolin., 1990, v. 31, № 2, p. 9-23.

7. Богачев В. И. Гауссовские меры. М.: Наука, 1997, 352 с.

8. Bogachev V.I. Differentiable measures and the Malliavin calculus. - J. Math. Sci. (New York), 1997, v. 87, № 4, p. 3577-3731.

9. Пугачев О.В. Формула Остроградского-Гаусса в бесконечномерном пространстве. - Матем. сб., 1998, т. 189, № 5, с. 115-128.

10. Пугачев О.В. Построение негауссовских поверхностных мер методом Маллявэна. - Матем. заметки, 1999, т. 65, № 3, с. 377-388.

11. Pugachev O.V. Tightness of Sobolev capacities in infinite dimensional spaces. - Infin. Dimens. Anal. Quantum Probab. Relat. Topics, 1999, v. 2, № 3, p. 427-440.

12. Röckner M., Schmuland B. Tightness of general $C_{1, p}$ capacities on Banach space. J. Funct. Anal., 1992, v. 108, № 1, p. 1-12.

13. Богачев В.И. Основы теории меры, т. 1, 2. М.-Ижевск: НИЦ «Регулярная и хаотическая динамика», 2006, 583 с.; 679 c.

14. Pugachev O. V. Capacities and surface measures in locally convex spaces. Preprint 06-064. Bielefeld, SFB701, 2006.

Поступила в редакцию 13.VI. 2007

(C) 2008 r.

\section{MA $C .^{*}$}

\section{INTRINSICALLY STATIONARY VARIOGRAMS IN SPACE AND TIME}

В статье обсуждается вопрос о том, когда произведение двух внутренне стационарных вариограмм структурных функций, а также степень внутренне стационарной вариограммы от пространственной и временной переменных остается вариограммой. Указывается несколько новых классов пространственно-временных вариограмм, а также приводятся степенные модели и модели с медленным затуханием ковариации.

* Department of Mathematics and Statistics, Wichita State University, Wichita, KS 67260-0033, USA; e-mail: cma@math.wichita.edu 